\title{
Rapanea ferruginea (Ruiz \& Pav.) Mez. (Myrsinaceae) como uma importante fonte alimentar para as aves em uma mata de galeria no interior do Estado de São Paulo
}

\author{
Márcia C. Pascotto \\ Departamento de Ciências Biológicas e da Saúde, Instituto Universitário do Araguaia, Universidade Federal de Mato Grosso. \\ Rodovia MT 100 km 3,5, 78698-000 Pontal do Araguaia, Mato Grosso, Brasil. E-mail: mcpascot@cpd.ufmt.br
}

\begin{abstract}
Rapanea ferruginea (Ruiz \& Pav.) Mez. (Myrsinaceae) as an important food resource for birds in a gallery forest in São Paulo, southeastern Brazil. Characteristic of humid habitats, Rapanea ferruginea produces globose drupes containing a single seed covered by a thin black-purplish pulp. The feeding behavior and the contribution of birds in dispersing $R$. ferruginea fruits was examined in a gallery forest edge in São Manuel $\left(22^{\circ} 43^{\prime} \mathrm{S}, 48^{\circ} 34^{\prime} \mathrm{W}\right)$, state of São Paulo, southeastern Brazil. In 38 hours and 40 minutes of focal observations carried out between October 20 to November 12, 2005, an amount of 11618 fruits were consumed by 31 bird species, probably due to both superabundance of food and exposure of fruits on tree. From the total, 25 species could be considered potential seed dispersers by swallowing the whole diaspore and having short and frequent visits. The most common feeder were Turdus amaurochalinus Cabanis, 1851, Dryocopus lineatus (Linnaeus, 1766) and Turdus leucomelas Vieillot, 1818, accounting for $43 \%$ of the total fruits consumed, being the former the most frequent in $R$. ferruginea. The birds involved removed fruits mainly by the picking technique, while hanging was the least employed, but for Vireo olivaceus (Vieillot, 1817) and D. lineatus. Aggressive interactions were minimal, being Mimus saturninus Hellmayr, 1903 the responsible for most interespecific encounters, in contrast to intraspecific actions dominated by $T$. amaurochalinus and $T$. leucomelas.
\end{abstract}

KEY WORDS. Avian frugivory; feeding behavior; seed dispersal.

RESUMO. Espécie típica de ambientes úmidos, Rapanea ferruginea produz diásporos globosos contendo uma única semente, envolta por um delgado pericarpo negro-arroxeado. Os comportamentos alimentares e a contribuição das aves na dispersão de sementes de $R$. ferruginea foram investigados em uma área de borda de mata de galeria, no município de São Manuel $\left(22^{\circ} 43^{\prime} \mathrm{S}, 4^{\circ} 34^{\prime} \mathrm{W}\right)$, estado de São Paulo, sudeste do Brasil. Em 38 horas e 40 minutos de observações realizadas entre 20 de outubro e 12 de novembro de 2005, foram consumidos 11618 frutos por 31 espécies de aves, sendo a alta taxa de remoção de diásporos provavelmente devido à superabundância de frutos e à exposição dos mesmos na planta. Das 31 espécies registradas, 25 foram consideradas como potenciais dispersores por engolirem o diásporo inteiro e por realizarem visitas freqüentes e de curta duração. Turdus amaurochalinus Cabanis, 1851 foi a espécie que mais se alimentou em $R$. ferruginea, seguida por Dryocopus lineatus (Linnaeus, 1766) e Turdus leucomelas Vieillot, 1818, responsáveis por $43 \%$ do total de frutos consumidos, sendo ainda a primeira a espécie mais freqüente. A técnica de captura de frutos mais empregada foi "picking", enquanto "hanging" foi a menos utilizada, mas predominante em Vireo olivaceus (Vieillot, 1817) e D. lineatus. Foram registrados poucos encontros agonísticos, sendo Mimus saturninus Hellmayr, 1903 a espécie responsável pelo maior número de encontros interespecíficos, enquanto que as interações intraespecíficas foram lideradas por $T$. amaurochalinus e por $T$. leucomelas. PALAVRAS-CHAVE. Comportamento alimentar; dispersão de sementes; frugivoria.

Um dos mecanismos pelos quais os vertebrados contribuem na dispersão de sementes é ingerindo frutos carnosos e defecando suas sementes em locais distantes da planta-mãe (STAPANIAN 1986), tendo as aves grande participação nesse processo. Assim, a frugivoria é o principal processo em que a regeneração natural das populações de plantas é fortemente dependente da disseminação de sementes por animais (JoRDANO
2000). Mas os fatores que influenciam a preferência dos frutos pelas aves envolvem tanto características dos próprios frutos como, por exemplo, grande produção de diásporos ou sementes (SNOw 1971, Herrera 1982), ou ainda cores contrastantes dos frutos maduros com frutos imaturos, folhas e pecíolos (STILES 1982). Para as aves frugívoras tropicais, Levey et al. (1984) salientaram que a preferência por frutos também pode ser deter- 
minada pela distância que uma ave percorre para procurar sua fonte alimentar e pela freqüência de suas visitas durante o forrageamento.

Espécies frutíferas de áreas abertas e de bordas ou fragmentos florestais geralmente têm taxas de remoção de frutos mais altas que aquelas do interior (THOMPSON \& Willson 1978), especialmente por aves generalistas. Outro fator que pode contribuir para aumentar a preferência dos frutos por aves é a frutificação em períodos de escassez de outros recursos alimentares (SNOW 1965).

Em florestas temperadas, parece ser comum a defesa de recursos alimentares por aves frugívoras (SNOw \& SNOw 1986), as quais empregam estratégias altamente desenvolvidas para impedirem outros indivíduos ou outras espécies de explorarem o mesmo recurso e conservando, assim, frutos para si mesmas por um longo tempo de escassez, especialmente no inverno. Já em florestas tropicais, apesar do grande número de aves frugívoras e da variedade de frutos disponíveis (SNOw 1981), há poucas evidências para defesa de frutos por um longo período de tempo. O que ocorrem são comportamentos agressivos esporádicos, tanto intra como interespecíficos, quando explorando a mesma fonte alimentar (e.g., Silva 1988, Francisco \& GaletTi 2001, Gondim 2001, Pascotto 2006). A ausência de defesa de recursos por aves frugívoras em florestas tropicais pode estar relacionada (I) ao curto período de frutificação da maioria das espécies, (II) à sucessão de amadurecimento de frutos por um longo período de tempo, (III) à ocorrência de árvores frutíferas de grande porte e com copa ampla ou (IV) à distribuição esparsa dos frutos nos ramos vegetativos (SNOw \& SNOw 1986).

Rapanea ferruginea (Ruiz \& Pav.) Mez. (Myrsinaceae) é uma árvore perenifólia, heliófila, higrófila e pioneira, característica de formações secundárias, como capoeiras e capoeirões, mas prefere encostas e beiras de córregos. Em determinado estágio da sucessão secundária da encosta atlântica, a canela-azeitona chega a ser a espécie predominante (Lorenzi 1992, Pinheiro \& CARMo 1993). Ocorre em todo o país, em quase todas as formações vegetais, sendo particularmente freqüente na floresta pluvial da encosta atlântica. Floresce duas vezes ao ano, podendo a mesma espécie estar com flores e frutos maduros na mesma árvore. Os frutos são pequenos (3-5 mm diâmetro), globosos e de coloração negro-arroxeada quando maduros, possuindo uma única semente e um pericarpo delgado (LoRENZI 1992).

Existem duas sinonímias na literatura botânica: Myrsine floculosa Mar. e Gaballeria ferruginea Ruiz \& Pav. Contudo, o nome latino válido é Rapanea ferruginea. Quanto aos nomes vulgares, a canela-azeitona é também conhecida por capororoca, azeitona-do-mato, camará, capororocaçu, capororoca-vermelha, pororoca e capororoca-mirim (LoRENZI 1992).

Pineschi (1990) realizou um estudo sobre o consumo de sementes de sete espécies de Rapanea em uma área de Mata Atlântica no Estado de São Paulo, incluindo R. ferruginea. Porém, o autor registrou apenas 12 espécies de aves que se alimentaram dos frutos da capororoca: Columba plumbea (Vieillot,
1818), Geotrygon violacea (Temminck, 1809) (Columbidae), Schizoeaca moreirae Cabanis, 1873 (Furnariidae), Piprites pileatus (Temminck, 1822) (Cotingidae), Chiroxiphia caudata (Shaw \& Nodder, 1793), Neopelma aurifrons (Wied-Neuwied, 1831) (Pipridae), Phyllomyias virescens (Temminck, 1824) (Tyrannidae), Platycichla flavipes (Vieillot, 1818), Turdus rufiventris Vieillot, 1818 (Turdinae), Cacicus chrysopterus (Vigors, 1825) (Icteridae), Chlorophonia cyanea (Thunberg, 1822) (Thraupinae) e Saltator maxillosus Cabanis, 1851 (Cardenalinae), mas não registrou os comportamentos alimentares, as freqüências de visitas e a taxa de consumo de frutos para cada espécie de ave em cada espécie de Rapanea amostrada. Assim, procurou-se avaliar mais detalhadamente a importância de Rapanea ferruginea na contribuição da dieta das aves e o papel destas como potenciais dispersores de suas sementes em uma área de mata de galeria. Durante as observações foram registradas: (I) as espécies de aves que se alimentaram dos frutos de Rapanea ferruginea; (II) o número de visitas de cada espécie; (III) o número de frutos consumidos; (IV) a duração das visitas; (V) a ocorrência e o número de comportamentos agonísticos; (VI) o comportamento de coleta dos frutos; e (VII) o número de frutos capturados em cada comportamento empregado.

\section{MATERIAL E MÉTODOS}

O estudo foi realizado em uma área de mata de galeria de propriedade particular no município de São Manuel $\left(22^{\circ} 43^{\prime}\right.$ S, $\left.48^{\circ} 34^{\prime} \mathrm{W}\right)$, Estado de São Paulo. A espécie vegetal amostrada encontra-se isolada a cerca de $50 \mathrm{~m}$ da borda da mata de galeria, que é margeada por áreas de pastagem e plantação de café.

As amostragens foram realizadas em dias consecutivos no período de 20 de outubro a 12 de novembro de 2005 , totalizando 38 horas e 40 minutos de observações. Todas as ocorrências observadas em um determinado período de tempo foram registradas, seguindo a metodologia ad libitum (AltMAN 1973) e também empregada em PAscotTo (2006). As observações diárias foram realizadas por um período mínimo de 60 min, compreendendo o horário das 6:30 às 19:10 h. Somente foram registradas as espécies que consumiram pelo menos um diásporo em cada visita, sendo desconsideradas as espécies que utilizaram a árvore para outros fins que não a alimentação.

Uma vez identificada a espécie de ave, seus comportamentos foram acompanhados e registrados desde sua chegada à árvore até sua saída, sendo a espécie novamente registrada decorridos cinco minutos desde sua última visita, exceto quando constatado tratar-se de indivíduos diferentes (dimorfismo sexual na coloração da plumagem) ou de espécies forrageando em grupos de dois ou mais indivíduos.

A freqüência de visitas para as espécies de aves registradas foi calculada seguindo a metodologia empregada por GoNDIM (2001) e PAscotTo (2006), na qual se dividiu o número total das visitas realizadas por cada espécie pelo número total de horas de observações. Já a taxa de consumo para cada espécie foi calculada dividindo-se o número de frutos consumidos por cada 
espécie pelo total de horas de observações (MotTa Junior 1990). As táticas de forrageamento foram definidas como: colher ("picking", P), alcançar ("reaching", R), dependurar ("hanging", Ha) e em vôo ("hovering", Ho), padronizadas de acordo com Moermond \& Denslow (1985) e utilizadas por Pascotto (2006) [ver descrição das categorias em Francisco \& Galetti (2001)].

A classificação das espécies quanto às dietas seguiu MотTA JUNIOR (1990). A classificação e a nomenclatura taxonômica das espécies seguiram Sick (1997).

O material coletado foi herborizado e incorporado à coleção de plantas do Herbário do Departamento de Ciências Biológicas da Universidade Estadual Paulista, campus de Bauru, Estado de São Paulo.

\section{RESULTADOS}

Após 38 horas e 40 minutos de observações, 31 espécies de aves representantes de três ordens e nove famílias consumiram diásporos de Rapanea ferruginea. A Ordem Passeriformes foi a mais representativa, com 27 espécies pertencentes a sete famílias, seguida das ordens Piciformes (3 espécies de Picidae) e Psittaciformes, sendo a única representante Brotogeris versicolurus (Statius Muller, 1776). Ao total, foram registradas 558 visitas e consumidos 11.618 frutos, resultando em 14,5 visitas/hora e 302,5 diásporos/hora (Tab. I).

Das 31 espécies registradas, 25 espécies (80,6\%) engolem a semente inteira, podendo ser consideradas potenciais dispersores de sementes de Rapanea ferruginea (Tab. I). Das demais espécies, apenas Ramphocelus carbo (Pallas, 1764), Sporophila caerulescens (Vieillot, 1817) e Cyclarhis gujanensis Baird, 1866 são consumidores de arilo, descartando a semente sobre a planta-mãe. As outras três espécies, Brotogeris versicolurus, Zonotrichia capensis Swainson, 1837 e Coereba flaveola (Cabanis, 1851), foram observadas tanto consumindo apenas o arilo e descartando as sementes, como também engolindo a semente inteira. Assim, R. carbo, S. caerulescens, C. gujanensis, B. versicolurus, Z. capensis e C. flaveola não podem ser consideradas potenciais dispersores de sementes de R. ferruginea (Tab. I).

Das espécies potencialmente dispersoras e com visitas freqüentes, Dacnis cayana Chubb, 1910, Thraupis sayaca (Linnaeus, 1766), Elaenia flavogaster (Thunberg, 1822), Pitangus sulphuratus (Cabanis \& Heine, 1859) e Myiarchus swainsoni Cabanis \& Heine, 1859 foram as espécies com menor duração de visitas em Rapanea ferruginea. Por outro lado, Dryocopus lineatus (Linnaeus, 1766) e Colaptes campestris (Vieillot, 1818) (Picidae) foram as espécies que mais tempo empregaram na alimentação. Apesar de Brotogeris versicolurus (Psittacidae) registrar a maior duração de visitas, este tempo foi ampliado pelo tempo gasto com outros comportamentos que não a alimentação (Tab. I).

Turdus amaurochalinus Cabanis, 1851 foi a espécie que mais visitou Rapanea ferruginea $(\mathrm{n}=75)$, seguida por Turdus leucomelas Vieillot, 1818 ( $\mathrm{n}=50)$, Tyrannus savana (Linnaeus, 1766) $(\mathrm{n}=$ 42) e Tyrannus melancholicus Vieillot, $1819(\mathrm{n}=41)$ (Tab. I).
Apesar de Cyclarhis gujanensis remover o fruto com o bico, ela empregava uma tática diferenciada na alimentação: voava para outro galho e prendia o fruto entre os artelhos, removendo pedaços do arilo com o bico, demandando um tempo maior no forrageamento.

Com relação às táticas de forrageamento, "picking" foi a mais empregada por todas as espécies, sendo utilizada exclusivamente por Saltator atricollis (Vieillot, 1817) para a remoção dos diásporos (Tab. II). As espécies de Tyrannidae, características por capturarem insetos em vôo, também coletaram boa parte dos diásporos consumidos em vôo ("hovering"). A tática "hanging" foi a menos utilizada pelas aves em $R$. ferruginea, sendo empregada principalmente por Vireo olivaceus (Vieillot, 1817) (Vireonidae) e Dryocopus lineatus (Picidae). Durante os períodos em que $D$. lineatus empregou a técnica "hanging" para sua alimentação, esta se agarrava firmemente com os artelhos aos ramos e permanecia por um determinado período de tempo com a região ventral voltada para cima, coletando os diásporos em tal posição. Assim que pousavam na árvore, os pica-paus empregavam comportamentos muito semelhantes àqueles utilizados para a captura de insetos em troncos, ou seja, utilizavam o estrato vertical para a procura dos frutos até chegarem aos ramos terminais.

Mimus saturninus Hellmayr, 1903 foi considerada a espécie mais agressora em Rapanea ferruginea. Foram registrados encontros agonísticos interespecíficos de M. saturninus, sempre individualmente, com: Tyrannus savana $(\mathrm{n}=4)$, Turdus amaurochalinus $(\mathrm{n}=3)$, Turdus rufiventris $(\mathrm{n}=2)$, Myiodinastes maculatus (Vieillot, 1819) ( $\mathrm{n}=2$ ), Tityra cayana (Swainson, 1837), Ramphocelus carbo, Turdus leucomelas, Empidonomus varius (Vieillot, 1818) e Pitangus sulphuratus, sendo registrado para cada uma destas últimas espécies apenas um comportamento agonístico. No entanto, M. saturninus não foi a espécie com maior número de visitas em $R$. ferruginea, sendo apenas a quinta espécie em número de visitas. Outros comportamentos agonísticos interespecíficos foram registrados, sempre individuais, com uma única ocorrência, sendo a primeira a espécie agressora: Thraupis sayaca e Tityra cayana, Turdus rufiventris e Camptostoma obsoletum (Temminck, 1824), Dryocopus lineatus e Brotogeris versicolurus, Elaenia flavogaster e Camptostoma obsoletum, Turdus leucomelas e Tyrannus savana, Turdus amaurochalinus e Sirystes sibilator (Vieillot, 1819), Myiodynastes maculatus e Tyrannus melancholicus, Turdus leucomelas e Colaptes campestris e entre Turdus leucomelas e Zonotrichia capensis. Já o maior número de comportamentos agonísticos intraespecíficos foi registrado para Turdus leucomelas $(\mathrm{n}=3)$ e T. amaurochalinus $(\mathrm{n}=2)$, sendo ainda registrado um único comportamento agonístico intraespecífico para Thraupis sayaca, Tyrannus melancholicus, Tyrannus savana e Myiarchus swainsoni.

Infrutescências e frutos imaturos foram observados sendo removidos por muitas espécies de aves, tais como: Colaptes campestris, Pitangus sulphuratus, Camptostoma obsoletum, Thraupis sayaca, Tangara cayana (Vieillot, 1819), Dacnis cayana, Mimus saturninus, Turdus rufiventris, T. leucomelas e Thlypopsis sordida 
Tabela I. Espécies de aves que se alimentaram em Rapanea ferruginea (Myrsinaceae) durante 38 h e 40 min de observações.

\begin{tabular}{|c|c|c|c|c|c|c|c|c|}
\hline Espécies & NV & $\begin{array}{l}\text { Duração das } \\
\text { visitas a }\end{array}$ & Dieta $^{\mathrm{b}}$ & $\begin{array}{c}\text { Frutos } \\
\text { consumidos }\end{array}$ & Frutos/visita $^{d}$ & Freq $^{\text {e }}$ & f & $\begin{array}{c}\text { Modo de } \\
\text { consumo }^{g}\end{array}$ \\
\hline \multicolumn{9}{|l|}{ Psittaciformes } \\
\hline \multicolumn{9}{|l|}{ Psittacidae } \\
\hline Brotogeris versicolurus (Statius Muller, 1776) & 5 & $718 \pm 560$ & FRU, $\mathrm{Pr}$ & 59 & $12 \pm 12,11$ & 0,13 & ND & ESI/MEA \\
\hline \multicolumn{9}{|l|}{ Piciformes } \\
\hline \multicolumn{9}{|l|}{ Picidae } \\
\hline Dryocopus lineatus (Linnaeus, 1766) & 12 & $352 \pm 186$ & INS & 1508 & $126 \pm 78,00$ & 0,31 & PD & ESI \\
\hline Colaptes campestris (Vieillot, 1818) & 11 & $256 \pm 151$ & INS & 652 & $59 \pm 33,29$ & 0,29 & PD & ESI \\
\hline Colaptes melanochloros (Malherbe, 1848) & 16 & $171 \pm 112$ & INS & 541 & $34 \pm 19,12$ & 0,42 & PD & ESI \\
\hline \multicolumn{9}{|l|}{ Passeriformes } \\
\hline \multicolumn{9}{|l|}{ Tyrannidae } \\
\hline Myiarchus swainsoni Cabanis \& Heine, 1859 & 23 & $87 \pm 48$ & INS & 138 & $6 \pm 2,88$ & 0,6 & PD & ESI \\
\hline Myiodynastes maculatus (Vieillot, 1819) & 22 & $126 \pm 134$ & ONI & 285 & $13 \pm 11,11$ & 0,58 & PD & ESI \\
\hline Pitangus sulphuratus (Cabanis \& Heine, 1859) & 16 & $87 \pm 51$ & ONI & 296 & $19 \pm 9,80$ & 0,42 & PD & ESI \\
\hline Megarhynchus pitangua (Linnaeus, 1766) & 4 & $94 \pm 31$ & ONI & 43 & $11 \pm 12,87$ & 0,1 & PD & ESI \\
\hline Tyrannus melancholicus Vieillot, 1819 & 41 & $125 \pm 73$ & INS & 810 & $20 \pm 13,53$ & 1,07 & PD & ESI \\
\hline Tyrannus savana (Linnaeus, 1766) & 42 & $135 \pm 105$ & INS & 727 & $17 \pm 11,07$ & 1,09 & PD & ESI \\
\hline Elaenia flavogaster (Thunberg, 1822) & 23 & $87 \pm 38$ & FRU & 205 & $9 \pm 5,55$ & 0,6 & PD & ESI \\
\hline Camptostoma obsoletum (Temminck, 1824) & 24 & $128 \pm 126$ & INS & 158 & $7 \pm 3,19$ & 0,63 & PD & ESI \\
\hline Empidonomus varius (Vieillot, 1818) & 27 & $107 \pm 85$ & INS & 248 & $9 \pm 8,98$ & 0,7 & PD & ESI \\
\hline Sirystes sibilator (Vieillot, 1819) & 4 & $159 \pm 111$ & INS & 38 & $10 \pm 8,19$ & 0,1 & PD & ESI \\
\hline Tityra cayana (Swainson, 1837) & 3 & $186 \pm 46$ & FRU & 28 & $9 \pm 2,31$ & 0,08 & PD & ESI \\
\hline \multicolumn{9}{|l|}{ Corvidae } \\
\hline Cyanocorax cristatellus (Temminck, 1823) & 2 & $200 \pm 141$ & ONI & 44 & $22 \pm 28,28$ & 0,05 & PD & ESI \\
\hline \multicolumn{9}{|l|}{ Emberizidae } \\
\hline \multicolumn{9}{|l|}{ Thraupinae } \\
\hline Piranga flava (Spix, 1825) & 3 & $163 \pm 21$ & ONI & 33 & $11 \pm 2,65$ & 0,08 & PD & ESI \\
\hline Thraupis sayaca (Linnaeus, 1766) & 33 & $81 \pm 58$ & ONI & 161 & $5 \pm 3,99$ & 0,86 & PD & ESI \\
\hline Tangara cayana (Vieillot, 1819) & 13 & $115 \pm 68$ & ONI & 74 & $6 \pm 3,20$ & 0,34 & PD & ESI \\
\hline Tersina viridis (Illiger, 1811) & 10 & $195 \pm 145$ & ONI & 112 & $11 \pm 5,29$ & 0,26 & PD & ESI \\
\hline Ramphocelus carbo Hellmayr, 1920 & 10 & $105 \pm 44$ & ONI & 63 & $6 \pm 2,50$ & 0,26 & ND & MEA \\
\hline Dacnis cayana Chubb, 1910 & 10 & $75 \pm 35$ & ONI & 39 & $4 \pm 2,77$ & 0,26 & PD & ESI \\
\hline \multicolumn{9}{|l|}{ Emberizinae } \\
\hline Zonotrichia capensis Swainson, 1837 & 8 & $51 \pm 27$ & GRA & 29 & $4 \pm 3,02$ & 0,21 & ND & ESI/MEA \\
\hline Sporophila caerulescens (Vieillot, 1817) & 1 & 40 & GRA & 1 & 1 & 0,03 & ND & MEA \\
\hline \multicolumn{9}{|l|}{ Cardenalinae } \\
\hline Saltator atricollis (Vieillot, 1817) & 1 & 165 & INS & 18 & 18 & 0,03 & PD & ESI \\
\hline \multicolumn{9}{|l|}{ Coerebinae } \\
\hline Coereba flaveola (Cabanis, 1851) & 1 & 80 & NEC & 4 & 4 & 0,03 & ND & ESI/MEA \\
\hline \multicolumn{9}{|l|}{ Muscicapidae } \\
\hline \multicolumn{9}{|l|}{ Turdinae } \\
\hline Turdus rufiventris Vieillot, 1818 & 22 & $119 \pm 85$ & ONI & 682 & $31 \pm 18,87$ & 0,58 & PD & ESI \\
\hline Turdus leucomelas Vieillot, 1818 & 50 & $117 \pm 81$ & $\mathrm{ONI}$ & 1378 & $28 \pm 16,64$ & 1,3 & PD & ESI \\
\hline Turdus amaurochalinus Cabanis, 1851 & 75 & $115 \pm 69$ & ONI & 2075 & $28 \pm 16,19$ & 1,95 & PD & ESI \\
\hline \multicolumn{9}{|l|}{ Mimidae } \\
\hline Mimus saturninus Hellmayr, 1903 & 34 & $110 \pm 49$ & $\mathrm{ONI}$ & 1062 & $31 \pm 15,15$ & 0,89 & PD & ESI \\
\hline \multicolumn{9}{|l|}{ Vireonidae } \\
\hline Vireo olivaceus (Vieillot, 1817) & 10 & $112 \pm 67$ & ONI & 95 & $10 \pm 4,40$ & 0,26 & PD & ESI \\
\hline Cyclarhis gujanensis Baird, 1866 & 2 & $146 \pm 49$ & INS & 12 & $6 \pm 7,07$ & 0,05 & ND & MEA \\
\hline
\end{tabular}

(NV) Número de visitas; ${ }^{a}$ duração média das visitas, em segundos (média \pm desvio padrão); ${ }^{b}$ (FRU) frugívoro, (GRA) granívoro, (INS)

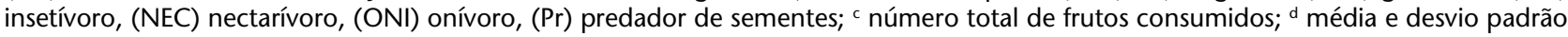
da quantidade de frutos consumidos por visita; ${ }^{e}$ freqüência de visitas em cada uma hora de observação; ${ }^{\dagger}$ espécies aqui consideradas como potenciais dispersoras de sementes de Rapanea ferruginea: (PD) potencial dispersora, (ND) não dispersora; ${ }^{g}$ (ESI) engole a semente inteira, (MEA) mandibula e engole somente o arilo, descartando a semente. 
Tabela II. Táticas de capturas dos frutos de Rapanea ferruginea (Myrsinaceae) por aves. (P) Picking, (R) reaching, ( $\mathrm{Ha}$ ) hanging, (Ho) hovering.

\begin{tabular}{|c|c|c|c|c|}
\hline Espécies & $P$ & $\mathrm{R}$ & $\mathrm{Ha}$ & Ho \\
\hline \multicolumn{5}{|l|}{ Psittaciformes } \\
\hline \multicolumn{5}{|l|}{ Psittacidae } \\
\hline Brotogeris versicolurus & 38 & 21 & 0 & 0 \\
\hline \multicolumn{5}{|l|}{ Piciformes } \\
\hline \multicolumn{5}{|l|}{ Picidae } \\
\hline Dryocopus lineatus & 962 & 336 & 210 & 0 \\
\hline Colaptes campestris & 583 & 69 & 0 & 0 \\
\hline Colaptes melanochloros & 458 & 83 & 0 & 0 \\
\hline \multicolumn{5}{|l|}{ Passeriformes } \\
\hline \multicolumn{5}{|l|}{ Tyrannidae } \\
\hline Myiarchus swainsoni & 26 & 2 & 1 & 109 \\
\hline Myiodynastes maculatus & 84 & 62 & 1 & 137 \\
\hline Pitangus sulphuratus & 164 & 68 & 0 & 64 \\
\hline Megarhynchus pitangua & 16 & 16 & 0 & 11 \\
\hline Tyrannus melancholicus & 629 & 75 & 0 & 106 \\
\hline Tyrannus savana & 531 & 76 & 0 & 120 \\
\hline Elaenia flavogaster & 130 & 48 & 4 & 23 \\
\hline Camptostoma obsoletum & 65 & 16 & 2 & 75 \\
\hline Empidonomus varius & 145 & 11 & 0 & 92 \\
\hline Sirystes sibilator & 10 & 0 & 0 & 28 \\
\hline Tityra cayana & 19 & 8 & 0 & 1 \\
\hline \multicolumn{5}{|l|}{ Corvidae } \\
\hline Cyanocorax cristatellus & 12 & 32 & 0 & 0 \\
\hline \multicolumn{5}{|l|}{ Thraupinae } \\
\hline Piranga flava & 15 & 18 & 0 & 0 \\
\hline Thraupis sayaca & 103 & 57 & 1 & 0 \\
\hline Tangara cayana & 42 & 32 & 0 & 0 \\
\hline Tersina viridis & 78 & 34 & 0 & 0 \\
\hline Ramphocelus carbo & 35 & 28 & 0 & 0 \\
\hline Dacnis cayana & 29 & 12 & 0 & 0 \\
\hline \multicolumn{5}{|l|}{ Turdinae } \\
\hline Turdus rufiventris & 577 & 105 & 0 & 0 \\
\hline Turdus leucomelas & 1217 & 153 & 0 & 8 \\
\hline Turdus amaurochalinus & 1831 & 233 & 0 & 11 \\
\hline \multicolumn{5}{|l|}{ Mimidae } \\
\hline Mimus saturninus & 910 & 152 & 0 & 0 \\
\hline \multicolumn{5}{|l|}{ Cardenalinae } \\
\hline Saltator atricollis & 18 & 0 & 0 & 0 \\
\hline \multicolumn{5}{|l|}{ Vireonidae } \\
\hline Vireo olivaceus & 18 & 51 & 23 & 3 \\
\hline Cyclarhis gujanensis & 11 & 1 & 0 & 0 \\
\hline \multicolumn{5}{|l|}{ Coerebinae } \\
\hline Coereba flaveola & 1 & 3 & 0 & 0 \\
\hline \multicolumn{5}{|l|}{ Emberizinae } \\
\hline Zonotrichia capensis & 26 & 3 & 0 & 0 \\
\hline Sporophila caerulescens & 1 & 0 & 0 & 0 \\
\hline
\end{tabular}

(Lafresnaye \& d'Orbegny, 1837), sendo que esta última não foi observada consumindo frutos maduros, embora tenha visitado a árvore por algumas vezes. No entanto, as infrutescências e os frutos imaturos consumidos não foram quantificados.

\section{DISCUSSÃO}

O alto consumo de diásporos de Rapanea ferruginea pode ser explicado tanto pela abundância de frutos (mais de 100 por ramo, segundo PINESCHI 1990) como pela facilidade de coleta dos mesmos pelas aves. Além dos frutos estarem localizados nas extremidades dos galhos, que são desprovidas de folhas, eles são pequenos (3-5 mm de diâmetro) e envoltos por um delgado pericarpo, que é fácil e rapidamente coletado pelas aves. Diferentemente, frutos encapsulados requerem, da maioria das aves, táticas diferenciadas e um tempo maior para a coleta, como observado em Alchornea glandulosa (Euphorbiaceae) por Pascotto (2006). Coates-Estrada \& Estrada (1988) demonstraram, por meio de experimentos, que sementes não encapsuladas (com ou sem arilo) são removidas mais rapidamente do que aquelas protegidas por cápsulas. Assim, a duração das visitas pelas aves e o tipo e a complexidade dos frutos estão correlacionados, como previsto por PratT \& STiles (1983).

A localização de $R$. ferruginea na borda da mata de galeria, onde há um predomínio de aves generalistas, também pode ter contribuído para o grande número de diásporos removidos, se comparado com os dados de Pineschi (1990), que registrou apenas 12 espécies de aves se alimentando em $R$. ferruginea no interior de uma área de Mata Atlântica no interior do Estado de São Paulo, corroborando Thompson \& Willson (1978). Das 12 espécies de aves registradas por Pineschi (1990), apenas Turdus rufiventris foi comum às duas áreas amostradas, o que reflete a diversidade da avifauna nesses ambientes.

Tityra cayana foi uma das três espécies frugívoras especialistas que se alimentaram em Rapanea ferruginea, mas responsável por um pequeno consumo de diásporos (média de $9 \pm 2,31$ ). Elaenia flavogaster, ao contrário, foi a espécie frugívora que mais visitou $R$. ferruginea, mas obteve uma taxa de consumo menor que Brotogeris versicolurus (Tab. I). Porém, E. flavogaster pode ser considerada a principal espécie frugívora potencialmente dispersora de $R$. ferruginea, uma vez que $B$. versicolurus é considerada como predadora de sementes (Howe \& Estabrook 1977, Sick 1997). Assim, a presença de frugívoros especialistas, apesar de baixa freqüência de visitas e do baixo consumo, também demonstra a importância da espécie vegetal na área e de sua contribuição na alimentação da avifauna local.

A maioria dos autores considera os Thraupinae como não dispersores de sementes (e.g., MotTa Junior \& Lombardi 1990, Gondim 2001), categorizando-os como consumidores de arilo. No entanto, apenas Ramphocelus carbo foi considerado como consumidor de arilo neste trabalho, sendo as demais espécies de Thraupinae potenciais dispersores de sementes de Rapanea ferruginea, o que pode estar relacionado ao pequeno tamanho dos frutos em relação ao tamanho do bico desses pássaros, como 
constatado por Levey (1986) em seus experimentos com tamanhos diferentes de sementes. Comparando-se os comportamentos alimentares de aves frugívoras em três espécies de plantas com diásporos de tamanhos diferentes, Levey (1986) verificou que aves grandes e mandibuladoras de diásporos, que possuem bicos maiores e com grande capacidade de abertura, tais como Pipra e Manacus, consumiram o maior número de sementes de pequeno porte, com cerca de 4,06 $\pm 0,3 \mathrm{~mm}$ de diâmetro, e com a menor taxa de descarte, quando comparadas às espécies mandibuladoras de tamanho corpóreo e com bico relativamente menor, como Euphonia e Tachyphonus. Apenas os Thraupinae com a maior angulação na abertura do bico, como Thraupis e Ramphocelus, conseguiram ingerir poucas sementes de tamanho maior, com cerca de 7,16 $\pm 55 \mathrm{~mm}$ de diâmetro. No entanto, algum outro fator pode estar influenciando a preferência de Ramphocelus carbo pelo arilo de Rapanea ferruginea, que não a relação tamanho do fruto versus tamanho do bico. Já SicK (1997) posiciona os Thraupinae entre as aves mais aptas à dispersão de sementes de plantas ornitocóricas, mas ressalta que Thraupis sayaca não é potencial dispersora de sementes por mandibular os diásporos e os deixarem cair sobre a planta mãe. Apesar da mandibulação dos diásporos ser um comportamento característico dos Thraupinae, especialmente de T. sayaca, não foram registrados descartes de sementes, sejam propositais ou acidentais, para esta espécie durante as observações, sendo T. sayaca considerada como potencial dispersora de sementes de $R$. ferruginea, bem como de Alchornea glandulosa (РАsсотTо 2006).

Apesar dos pica-paus serem basicamente insetívoros, a inclusão de frutos em sua dieta vem sendo registrada em alguns trabalhos, como em Howe (1977), Howe \& De STEven (1979), Coates-Estrada \& Estrada (1988), Argel-de-Oliveira (1992), Marcondes-Machado et al. (1994), Figueiredo et al. (1995) e FranCisco \& GALETTI (2001), como também constatado neste estudo. As três espécies de pica-paus registradas foram responsáveis, juntas, por cerca de $23 \%$ do consumo total de diásporos de Rapanea ferruginea (Tab. I).

Brotogeris versicolurus (Psittacidae) e Zonotrichia capensis (Emberizinae), apesar de terem sido observadas ingerindo a semente inteira por algumas vezes, não podem ser consideradas como potenciais dispersores de sementes, uma vez que Howe \& Estabroor (1977) consideraram que os Psittacidae, Fringillidae (= Emberizidae) e Columbidae digerem as sementes. No entanto, fazem-se necessárias investigações futuras para saber se as sementes de $R$. ferruginea passam íntegras ou não pelo trato digestivo dessas espécies.

Apesar de Saltator atricollis pertencer a um grupo de aves com hábitos alimentares granívoros, a subfamília Cardenalinae, esta espécie pode ser considerada como potencial dispersora de sementes de $R$. ferruginea por ingerir a semente inteira, como também constatado por Argel-de Oliveira (1992). Além disso, Silva (1988) e PINESCHI (1990) realizaram experimentos com as sementes eliminadas nas fezes dessas duas espécies e constataram que as mesmas passaram íntegras pelo trato digestivo.
A tática de Cyclarhis gujanensis empregada na alimentação dos frutos de Rapanea ferruginea é semelhante àquela empregada em Alchornea glandulosa (РА⿱宀八 (Pото 2006), apesar da diferença de tamanho dos diásporos das duas plantas ser considerável. Após retirar a semente da planta, C. gujanensis voa para outro galho, prende a semente entre os artelhos e remove pedaços do arilo por meio de bicadas, descartando a semente em seguida sobre a planta-mãe. A tática de segurar as sementes com os pés e de remover pedaços do arilo das sementes de $R$. ferruginea e de A. glandulosa (PAscotTo 2006) por meio de bicadas é a mesma utilizada na captura e na alimentação de insetos (SICK 1997), já que C. gujanensis é primariamente insetívora.

A ocorrência de comportamentos agonísticos em Rapanea ferruginea foi pouco observada $(\mathrm{n}=34)$, como também para Alchornea glandulosa $(\mathrm{n}=23$, РАscotTo 2006), Cereus peruviano ( $\mathrm{n}=10$, Silva 1988), Rapanea lancifolia $(\mathrm{n}=11$, Francisco \& GALETTI 2001) e Trichilia spp. ( $\mathrm{n}=29$, Gondim 2001), se comparada com a ocorrência de interações em Dunalia arborescens (n $=58$, CRuz 1981) e Cabralea canjerana $(\mathrm{n}=110$, PIzo 1997). Tal resultado pode estar relacionado tanto à abundância de diásporos de $R$. ferruginea como à disponibilidade de outros recursos alimentares na área, não tendo as espécies que defender sua fonte alimentar para conservá-la por um longo período de escassez de recursos. Outros dois fatores que provavelmente podem ter influenciado as poucas interações agonísticas em $R$. ferruginea são o seu curto período de frutificação (cerca de um mês), embora frutifique duas vezes por ano, e o predomínio de espécies generalistas na área.

A abundância de uma determinada espécie de ave em uma região não resulta, necessariamente, no maior número de visitas e/ou de consumo de frutos em uma dada espécie vegetal. Na área estudada, Thraupis sayaca é a espécie mais abundante (obs. pess.) e foi a que mais se alimentou e visitou Alchornea glandulosa (Pascotтo 2006), mas foi apenas a sexta espécie em número de visitas em Rapanea ferruginea, com uma pequena taxa de consumo. Vale ressaltar que as duas espécies vegetais, $A$. glandulosa e $R$. ferruginea, encontram-se na mesma propriedade particular e ambas localizam-se na borda da mesma mata de galeria, com apenas cerca de $500 \mathrm{~m}$ de distância entre si. Assim, outros fatores que não a abundância de uma dada espécie parece determinar o consumo de diásporos, como a preferência alimentar das espécies de aves e a oferta de outros recursos alimentares em uma determinada estação.

\section{AGRADECIMENTOS}

A Osmar Cavassan, Departamento de Ciências Biológicas, Universidade Estadual Paulista, campus de Bauru, pela identificação da espécie vegetal, à Ana Lúcia Ferro, pelo auxílio em algumas observações em campo, e a dois revisores anônimos.

\section{REFERÊNCIAS BIBLIOGRÁFICAS}

Altmann, J. 1973. Observational study of behavior: sampling methods. Behaviour 49: 227-267. 
Argel-de-Oliveira, M.M. 1992. Comportamento alimentar de aves em Trichilia micrantha Benth. (Meliaceae) na Serra dos Carajás, Pará. Boletim do Museu Paraense Emílio Goeldi, Série Zoologia, 8: 305-313.

Coates-Estrada, R. \& A. Estrada. 1988. Frugivory and seed dispersal in Cymbopetalum baillonii (Annonaceae) at Los Tuxtlas, Mexico. Journal of Tropical Ecology 4: 157-172.

Cruz, A. 1981. Bird activity and seed dispersal of a montane forest tree (Dunalia arborescens) in Jamaica. Biotropica 34: 34-44.

Figueiredo, R.A.; J.C. Motta Junior \& L.A.S. Vasconcellos. 1995. Pollination, seed dispersal, seed germination and establishment of seedlings of Ficus microcarpa, Moraceae, in southeastern Brazil. Revista Brasileira de Biologia 55: 233-239.

Francisco, M.R. \& M. Galetti. 2001. Frugivoria e dispersão de sementes de Rapanea lancifolia (Myrsinaceae) por aves numa área de cerrado do Estado de São Paulo, sudeste do Brasil. Ararajuba 9 (1): 13-19.

Gondim, M.J.C. 2001. Dispersão de sementes de Trichilia spp. (Meliaceae) por aves em um fragmento de mata mesófila semidecídua, Rio Claro, SP, Brasil. Ararajuba 9 (2): 101-112.

Herrera, C.M. 1982. Defense of ripe fruit from pests: its significance in relation to plant-disperser interactions. The American Naturalist 120: 218-241.

Howe, H.F. 1977. Bird activity and seed dispersal of a tropical wet forest tree. Ecology 58: 539-550.

Howe, H.F. \& D. De STEven. 1979. Fruit production, migrant bird visitation, and seed dispersal of Guarea glabra in Panama. Oecologia 39: 185-196.

Howe, H.F. \& G.F. EsтавRоOK. 1977. On the intraspecific competition for avian dispersers in tropical trees. The American Naturalist 111: 817-832.

Jordano, P. 2000. Fruits and frugivory, p. 125-165. In: M. FENNER (Ed.). Seeds: the ecology of regeneration in plant communities. New York, CABI Publishing, $2^{\text {nd }}$ ed., 448p.

LeVEY, D.J. 1986. Methods of seed processing by birds and seed deposition patterns, p. 147-158. In: A. EstradA \& T.H. Fleming (Eds). Frugivores and seed dispersal. Dordrecht, W. Junk Publishers, 392p.

Levey, D.J.; T.C. Moermond \& J.S. Denslow. 1984. Fruit choice in neotropical birds: the effects of distance between fruits on preference patterns. Ecology 65: 844-850.

Lorenzi, H. 1992. Árvores brasileiras. Nova Odessa, Plantarum, $352 \mathrm{p}$.

Marcondes-Machado, L.O.; S.J. Paranhos \& Y.M. Barros. 1994. Estratégias alimentares de aves na utilização de frutos de Ficus microcarpa (Moraceae) em uma área antrópica. Iheringia, Série Zoologia, 77: 57-62.

Moermond, T.C. \& J.S. Denslow 1985. Neotropical avian frugivores: patterns of behavior, morphology, and nutrition, with consequences for fruit selection. Ornithological Monography 36: 865-897.

Motta Junior, J.C. 1990. Estrutura trófica e composição das avifaunas de três hábitats terrestres na região central do estado de São Paulo. Ararajuba 1: 65-71.

MotTA Junior, J.C. \& J.A. Lombardi. 1990. Aves como dispersores de copaíba (Copaifera langsdorffii, Caesalpiniaceae) em São Carlos, estado de São Paulo. Ararajuba 1: 105-106.

Pascotto, M.C. 2006. Avifauna dispersora de sementes de Alchornea glandulosa (Euphorbiaceae) em uma área de mata ciliar no estado de São Paulo. Revista Brasileira de Ornitologia 14 (3): 291-296.

Pineschi, R.B. 1990. Aves como dispersores de sete espécies de Rapanea (Myrsinaceae) no maciço do Itatiaia, Estados do Rio de Janeiro e Minas Gerais. Ararajuba 1: 73-78.

Pinheiro, A.L. \& A.P.T. Carmo. 1993. Contribuição ao estudo tecnológico da canela-azeitona, Rapanea ferruginea (Ruiz e Pav.) Mez, uma espécie pioneira. I. Características anatômicas da madeira. Ciência Flor 3 (1): 121-145.

Pizo, M.A. 1997. Seed dispersal and predation in two populations of Cabralea canjerana (Meliaceae) in the Atlantic Forest of southeastern Brazil. Journal of Tropical Ecology 13: 559578.

Pratt, T.K. \& E.W. STILEs. 1983. How long fruit-eating birds stay in the plants where they feed: implications for seed dispersal. The American Naturalist 122: 797-805.

SICK, H. 1997. Ornitologia Brasileira. Rio de Janeiro, Editora Nova Fronteira, 912p.

Silva, W.R. 1988. Ornitocoria em Cereus peruvianus (Cactaceae) na Serra do Japi, estado de São Paulo. Revista Brasileira de Biologia 48: 381-389.

SNow, D.W. 1965. A possible selective factor in the evolution of fruiting seasons in tropical forest. Oikos 15: 274-281.

SNow, D.W. 1971. Evolutionary aspects of fruit-eating by birds. The Ibis 113: 194-202.

SNow, D.W. 1981. Tropical frugivorous birds and their food plants: a world survey. Biotropica 13: 1-14.

SNow, D.W. \& B.K. SNOw. 1986. Some aspects of avian frugivory in a north temperate area relevant to tropical forest, p. 159164. In: A. Estrada \& T.H. Fleming (Eds). Frugivores and seed dispersal. Dordrecht, W. Junk Publishers, 392p.

Stapanian, M.A. 1986. Seed dispersal by birds ans squirrels in the deciduous forests of the United States, p. 225-236. In: A. Estrada \& T.H. Fleming (Eds). Frugivores and seed dispersal. Dordrecht, W. Junk Publishers, 392p.

Stiles, E.W. 1982. Fruit flags: two hypotheses. The American Naturalist 120: 500-509.

Thompson, J.N. \& M.F. Willson. 1978. Disturbance and the dispersal of fleshy fruits. Science 20: 1161-1163.

Recebido em 16.I.2007; aceito em 22.VIII.2007. 